\title{
Teores e estoque de carbono em frações lábeis e recalcitrantes da matéria orgânica do solo sob integração lavoura-pecuária no bioma Cerrado
}

\section{Content and carbon stocks in labile and recalcitrant organic matter of the soil under crop-livestock integration in Cerrado ${ }^{1}$}

\author{
Itaynara Batista ${ }^{2}$; Marcos Gervasio Pereira ${ }^{3}$; Maria Elizabeth Fernandes Correia ${ }^{4}$; \\ Wanderlei Bieluczyk ${ }^{5}$; Jolimar Antônio Schiavo ${ }^{6}$; Janaína Ribeiro Costa Rows ${ }^{4}$
}

\section{Resumo}

O estudo da matéria orgânica e seus compartimentos, e sua relação com o manejo, visa desenvolver estratégias para o aumento dos seus teores em solos cultivados e melhor compreensão de sua dinâmica. Este trabalho teve como objetivo avaliar as frações granulométricas da matéria orgânica do solo e seus respectivos estoques de carbono em diferentes coberturas do solo em sistema integração lavourapecuária e vegetação nativa de Cerrado. O trabalho foi desenvolvido na fazenda Cabeceira, Maracaju MS, as áreas amostradas possuem o seguinte histórico: soja/milho+braquiária/algodão/aveia+pasto/soja/ formação do pasto/pasto, as amostragens foram realizadas em duas épocas, seca (maio/2009) e chuvosa (março/2010), na época seca, as culturas presentes eram: pasto, milho+braquiária e algodão e na época chuvosa eram milho, algodão e soja, assim, as áreas nas duas épocas de avaliação foram: pasto/milho, milho+braquiária/algodão, algodão/soja e uma área nativa de Cerradão. Foi realizada a determinação do complexo sortivo, análise granulométrica, densidade do solo, carbono orgânico, fracionamento granulométrico da matéria orgânica do solo com a quantificação do carbono orgânico particulado (COp) e do carbono orgânico associado aos minerais (COam). Foi também quantificado o estoque de carbono e das frações granulométricas. A área de pasto/milho proporcionou maior estoque de carbono na fração particulada na camada superficial do solo. A área de algodão/soja, devido a sua textura mais arenosa, foi a que apresentou maiores perdas de carbono. Devido às áreas possuírem o mesmo histórico, o estoque da fração mais humificada não se mostrou sensível às variações de cobertura. A fração COp apresenta-se mais sensível às diferentes coberturas do solo e sazonalidade.

Palavras-chave: Fracionamento granulométrico, sazonalidade, manejo

\footnotetext{
Abstract

The study of organic matter and its compartments and their relationship with management, aims to develop strategies for increasing their levels in soils and better understanding of its dynamics. This work aimed to evaluate the fractions of soil organic matter and their carbon stocks in different soil cover system in crop-livestock integration and native Cerrado vegetation. The study was conducted at

${ }^{1}$ Parte da dissertação de Mestrado da primeira autora apresentada ao Curso de Pós-Graduação em Agronomia, Ciência do Solo, CPGA-CS, Universidade Federal Rural do Rio de Janeiro, URRJ, Rio de Janeiro. Apoio CNPq.

${ }^{2}$ Discente de Doutorado em Agronomia em Agricultura, Universidade Estadual Paulista, Faculdade de Ciências Agronômicas, Campus de Botucatu, UNESP, Botucatu, SP. E-mail: itaynarabatista@yahoo.com.br

${ }^{3}$ Prof. Associado IV do Dept ${ }^{\circ}$ de Solos da UFRRJ, Seropédica, RJ. E-mail: gervasio@ufrrj.br

${ }^{4}$ Pesquisadores da Embrapa Agrobiologia, Seropédica, RJ. E-mail: elizabeth.correia@embrapa.br; janaina@cnpab.embrapa.br

${ }_{5}^{5}$ Discente de Mestrado do CPGA-CS, Dept ${ }^{\circ}$ de Solos, UFRRJ, RJ. E-mail: wanderleibieluczyk@gmail.com

${ }^{6}$ Prof. Adjunto IV da Universidade Estadual de Mato Grosso do Sul, UEMS, Aquidauana, MS. E-mail: schiavo@uems.br

* Autor para correspondência
} 
the farm Cabeceira, Maracajú - MS, sample area have the following history: soybean/corn + brachiaria/ cotton/oat + pasture/soybean/formation of pasture/grazing, sampling was carried out in two seasons, dry (May/2009) and rainy (March 2010), in the dry season, crops present were: pasture, corn and cotton + brachiaria and in the rainy season were corn, cotton and soybeans, so the areas in the two evaluation periods were: pasture / maize + brachiaria / cotton, cotton / soybean area and a native of Savanna. Was performed to determine the exchangeable cations, particle size analysis, bulk density, organic carbon, particle size fractionation of organic matter of the soil with the quantification of particulate organic carbon (POC) and organic carbon associated with minerals (OCam). Was also quantified the carbon stock and size fractions. The area of pasture / maize showed higher carbon stock in the particulate fraction in the topsoil. The area of cotton / soy due to its lower clay, showed the greatest loss of carbon. Because of the areas have the same history, the stock of more recalcitrant fraction was not sensitive to variations in coverage. The POC fraction appears more sensitive to different soil covers and seasonality.

Key words: Particle size fractionation, seasonality, management

\section{Introdução}

O Cerrado é o segundo maior bioma brasileiro e representa cerca de $12,4 \%$ do território do país (IBGE, 2010). Transformações do uso da terra no Cerrado tiveram início na década de 70 , mediante a adoção de técnicas inadequadas de manejo do solo que causaram rápida redução no conteúdo de nutrientes e da matéria orgânica (RESK et al., 2008). Nesse contexto, o sistema de plantio direto (SPD) surgiu como alternativa para a redução nas operações de preparo do solo e contribuir para a formação e manutenção da cobertura morta (CRUZ et al., 2006). Outro entrave para a produção no Cerrado está relacionado à grande quantidade de pastagens degradadas, que de acordo com Silva et al. (2004), apresentam área superior a 50 milhões de hectares sob condições de baixa fertilização e manejo inadequado. Diante desse contexto, surgiu a integração lavoura-pecuária (ILP), que permitiu a utilização de sistemas de exploração em esquemas de rotação, onde se alternam anos ou períodos de pecuária com a produção de grãos ou fibras (MACEDO, 2009). Neste sistema o uso da terra é alternado, no tempo e no espaço, entre lavoura e pecuária (VILELA et al., 2011)

O consórcio de culturas de grãos com forrageiras é adotado para antecipar o estabelecimento das pastagens e melhorar a cobertura de solo para o plantio direto, pois este se apresenta como uma importante alternativa para a produção de massa vegetal sobre o solo. De modo geral, pastagens bem manejadas, têm potencial para aumentar o teor de carbono do solo, pois em áreas de pastagens a deposição de dejetos pelos animais pode constituirse em importante fator de reciclagem e de concentração de carbono e nitrogênio no solo. Com a presença do subsistema animal, são ampliadas as relações entre os compartimentos da matéria orgânica, tornando-as mais complexas (SALTON et al., 2005; VILELA et al., 2011).

A matéria orgânica do solo (MOS) apresenta potencial para ser utilizada como indicador da qualidade do solo (DORAN; PARKIN, 1994). É um dissipador temporário de nutrientes, provome melhorias na agregação e consquentemente na infiltração e retenção da água no solo e reduz os processos erosivos. A quantificação da MOS é portanto, um passo importante para a avaliação geral da qualidade do solo (GREGORICH et al., 1994). O acúmulo da MOS é altamente sensível ao sistema de manejo adotado, sendo este acúmulo também modificado por fatores abióticos como a precipitação e a temperatura (FRANZLUEBBERS, 2002), demonstrando o efeito da sazonalidade na sua dinâmica.

O carbono $(\mathrm{C})$ pode acumular-se em frações lábeis ou estáveis da MOS, o que pode ter implicações na retenção de $\mathrm{C}$ atmosférico e alterações nas propriedades físicas, químicas e biológicas dos solos (BAYER et al., 2004). As frações da MOS, presentes em diferentes compartimentos, podem apresentar velocidades de decomposição 
distintas, dependendo da sua localização física no solo (FREIXO; CANELLAS; MACHADO, 2002a). A MOS pode ser estratificada através de diferentes tipos de fracionamento, podendo estes serem classificados como químicos ou físicos. Cambardella e Elliot (1992) apresentaram um método simples que combina dispersão química com uma subsequente separação com base no diâmetro das partículas, sendo este denominado de fracionamento granulométrico. Através desta análise é possível quantificar os teores de carbono orgânico com tamanho da fração areia (diâmetro $\geq 53 \mu \mathrm{m}$ ) (denominado de carbono orgânico particulado COp) e aquele associado as frações silte e argila

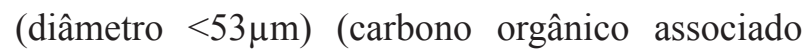
aos minerais - Coam). Segundo os autores, a capacidade de estimar quantitativamente frações da MOS é importante para a compreensão da dinâmica da MOS no manejo de sistemas intensivos.

O COp apresenta elevada sensibilidade ao manejo, podendo ser mais sensível que o carbono orgânico (C org), a análise dessa fração possibilita que modificações nos sistemas de manejo sejam prontamente adotadas antes que a qualidade do solo seja impactada de forma severa (CONCEIÇÃO et al., 2005). O Coam apresenta uma ciclagem lenta, no que se refere à sua formação e decomposição (BAYER et al., 2004), é a fração mais estável não apresentando sensibilidade imediata a alterações em práticas de manejo do solo, portanto, constituise no estoque de carbono à médio e longo prazo (SALTON et al., 2005).

Em sistemas agrícolas, os estoques de C org são também influenciados pelo manejo adotado, com a adição diferenciada de resíduos ao solo devemse ter diferentes estoques de carbono (SOUZA et al., 2009; COSTA et al., 2008). Um manejo do solo inadequado resulta em um rápido declínio do estoque de carbono, promovendo aumento de emissões de gás carbônico.

O estudo da matéria orgânica, em seus diversos compartimentos, bem como sua relação com o manejo, visa desenvolver estratégias para uma utilização sustentável dos solos, com vistas em reduzir o impacto das atividades agrícolas sobre o ambiente, principalmente na região do Cerrado, onde as altas temperaturas podem contribuir para um declínio acelerado dos estoques de C (FREIXO et al., 2002b). Diante do exposto, este trabalho teve por objetivo avaliar as frações granulométricas da matéria orgânica do solo e seus respectivos estoques de carbono em diferentes coberturas do solo em sistema integração lavoura-pecuária e vegetação nativa de Cerrado.

\section{Material e Métodos}

A área de estudo localiza-se na Fazenda Cabeceira, Maracaju - MS, latitude 2136'52" S e longitude $55^{\circ} 10^{\prime} 06^{\prime \prime} \mathrm{W}$, em uma altitude de 384 metros. O clima da região é tropical com valores médios de temperaturas mínimas de $15^{\circ} \mathrm{C}$ e máximas de $33{ }^{\circ} \mathrm{C}$, e precipitação média anual de 1300 a 1500 $\mathrm{mm}$.

As amostragens foram realizadas na Fazenda Cabeceira, que foi uma das pioneiras na implantação da ILP no Cerrado em 1989, sendo inicialmente introduzida a rotação de soja com pasto em SPD e em 2003 implantado o sistema que funciona atualmente, consistindo em pasto, soja e algodão como culturas de verão e pasto, milho+braquiária e aveia+pasto como culturas de inverno. As áreas possuem o seguinte histórico de culturas: soja/ milho+braquiária/algodão/aveia+pasto/soja/ formação do pasto/pasto. A carga animal nas áreas de pasto é de 3,5 cab ha-1.

Foram realizadas duas amostragens, sendo a primeira na época seca (maio de 2009) onde as culturas que se encontravam na área eram: pasto, cujo talhão avaliado possuía 43,04 ha; milho+braquiária em estádio vegetativo em talhão de 51 ha; e algodão recém colhido em talhão de 57,29 ha, ambos conduzidos no sistema integração lavoura-pecuária. Também foi avaliada uma área de Cerradão, que foi utilizada como referência. A segunda amostragem 
foi realizada na época chuvosa (março de 2010) e as culturas presentes no momento da amostragem eram milho, que estava consorciado com braquiária e algodão ambos em estádio vegetativo e soja recém colhida. As áreas nas duas épocas de avaliação foram: Cerradão, pasto/milho, milho+braquiária/ algodão e algodão/soja (Tabela 1). A área de pasto/ milho corresponde à área de pasto na época seca e milho na época chuvosa; a área de milho+braquiária/ algodão corresponde à área de milho+braquiária na época seca e algodão na época chuvosa, o solo nessas áreas foi identificado como Latossolo Vermelho. A área de algodão/soja encontrava-se com algodão na época seca e soja na época chuvosa, nessa área o solo foi classificado como Latossolo VermelhoAmarelo (EMBRAPA, 2006).

Tabela 1. Áreas avaliadas nas épocas seca (maio/2009) e época chuvosa (março/2010).

\begin{tabular}{ccc}
\hline & Uso anterior & Uso no momento da amostragem \\
\hline Áreas & Época seca & \\
\hline Cerradão & Cerradão & Cerradão \\
pasto/milho & Pasto & Pasto \\
milho+braquiária/algodão & Soja & milho + braquiária \\
algodão/soja & algodão & \\
& milho+braquiária & Cerradão \\
& Época chuvosa & milho \\
\hline Cerradão & Cerradão & algodão \\
pasto/milho & Soja & soja \\
\hline milho+braquiária/algodão & milho+braquiária & aveia+pasto \\
algodão/soja & &
\end{tabular}

Fonte: Elaboração dos autores.

Em cada uma das áreas, foram abertas seis mini-trincheiras espaçadas $50 \mathrm{~m}$ entre si nos talhões, nestas, foram coletadas amostras nas profundidades de 0-10, 10-20 e 20-30 cm. Após a coleta as amostras foram secas ao ar, destorroadas e passadas por peneira de 2,00 $\mathrm{mm}$, obtendo-se assim a terra fina seca ao ar (TFSA). Neste material foi realizada a análise granulométrica (DAY, 1965), caracterização do complexo sortivo (Tabela 2), densidade do solo (Ds), segundo Embrapa (1997), a determinação do C org (YEOMANS; BREMNER, 1988) e fracionamento granulométrico da matéria orgânica para obtenção das frações do carbono orgânico (COp e COam) (CAMBARDELLA;
ELLIOT, 1992), para isso, cerca de $20 \mathrm{~g}$ de solo e $60 \mathrm{~mL}$ de solução de hexametafosfato de sódio $(5 \mathrm{~g}$ $\left.\mathrm{L}^{-1}\right)$ foram agitados durante 15 horas em agitador horizontal. Em seguida, a suspensão foi passada em peneira de $53 \mu \mathrm{m}$ com auxílio de jato de água. O material que ficou retido na peneira, é o COp, do tamanho da fração areia, que foi seco em estufa à $50^{\circ} \mathrm{C}$, quantificado em relação a sua massa, moído em gral de porcelana e analisado em relação ao teor de C org segundo Yeomans e Bremner (1988). O material que passou pela peneira de $53 \mu \mathrm{m}$, consistiu no COam das frações silte e argila, e foi obtido por diferença entre o COT e COp. 
Tabela 2. Atributos químicos de amostras de solo nas profundidades 0-10, 10-20 e 20-30 cm.

\begin{tabular}{|c|c|c|c|c|c|c|c|c|c|c|}
\hline \multirow[t]{2}{*}{ Área } & \multicolumn{2}{|c|}{$\mathrm{pH}\left(\mathrm{H}_{2} \mathrm{O}\right)$} & \multicolumn{2}{|c|}{$\mathrm{Ca}$} & \multicolumn{2}{|c|}{$\mathrm{Mg}$} & \multicolumn{2}{|c|}{$\mathrm{Na}$} & \multicolumn{2}{|c|}{$\mathrm{K}$} \\
\hline & $\mathrm{S}$ & $\mathrm{C}$ & S & $\mathrm{C}$ & $\mathrm{S}$ & $\mathrm{C}$ & $\mathrm{S}$ & $\mathrm{C}$ & $\mathrm{S}$ & $\mathrm{C}$ \\
\hline Cerradão & 5,75 & 5,23 & 10,05 & 3,15 & 5,33 & 2,23 & 0,09 & 0,03 & 0,16 & 0,21 \\
\hline pasto/milho & $5,69 \mathrm{Ba}$ & $5,85 \mathrm{Aa}$ & 7,50 Aa & $4,86 \mathrm{Ab}$ & $3,75 \mathrm{Aa}$ & $3,4 \mathrm{Aa}$ & $0,09 \mathrm{Aa}$ & $0,04 \mathrm{ABb}$ & $0,19 \mathrm{Ab}$ & $0,63 \mathrm{Aa}$ \\
\hline $\begin{array}{c}\text { milho+braquiária/ } \\
\text { algodão }\end{array}$ & $5,71 \mathrm{Ba}$ & $5,42 \mathrm{Bb}$ & $4,62 \mathrm{Ba}$ & $3,2 \mathrm{Ab}$ & $3,38 \mathrm{Aa}$ & $3,34 \mathrm{Aa}$ & $0,09 \mathrm{Aa}$ & $0,03 \mathrm{Bb}$ & $0,26 \mathrm{Ab}$ & $0,70 \mathrm{Aa}$ \\
\hline algodão/soja & $6,41 \mathrm{Aa}$ & $5,66 \mathrm{ABb}$ & 7,05 Aa & $4,15 \mathrm{Ab}$ & $3,57 \mathrm{Aa}$ & $2,03 \mathrm{Ab}$ & $0,09 \mathrm{Aa}$ & $0,04 \mathrm{Ab}$ & $0,20 \mathrm{Ab}$ & $0,41 \mathrm{Aa}$ \\
\hline \multirow[t]{3}{*}{ Área } & \multirow{2}{*}{\multicolumn{2}{|c|}{ SB }} & \multicolumn{2}{|c|}{$\mathrm{H}+\mathrm{Al}$} & \multicolumn{2}{|c|}{$\mathrm{T}$} & \multirow{2}{*}{\multicolumn{2}{|c|}{$\mathrm{V} \%$}} & \multirow{2}{*}{\multicolumn{2}{|c|}{$\begin{array}{c}\mathrm{P} \\
\mathrm{mg} \mathrm{kg-1}\end{array}$}} \\
\hline & & & $---\mathrm{cmol}_{\mathrm{c}}$ & $\mathrm{kg}^{-1}$ solo-- & 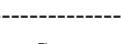 & --------- & & & & \\
\hline & $\mathrm{S}$ & $\mathrm{C}$ & S & $\mathrm{C}$ & $\mathrm{S}$ & $\mathrm{C}$ & $\mathrm{S}$ & $\mathrm{C}$ & $\mathrm{S}$ & $\mathrm{C}$ \\
\hline Cerradão & 15,63 & 5,62 & 6,5 & 8,69 & 22,14 & 14,31 & 70 & 39 & 1,99 & 0,28 \\
\hline pasto/milho & $11,53 \mathrm{Aa}$ & $8,92 \mathrm{Ab}$ & $3,88 \mathrm{Aa}$ & 4,65 $\mathrm{Aa}$ & $15,41 \mathrm{Aa}$ & $13,57 \mathrm{Ab}$ & $75 \mathrm{Ba}$ & $66 \mathrm{ABb}$ & $23,94 \mathrm{Aa}$ & $22,28 \mathrm{ABa}$ \\
\hline milho/algodão & $8,36 \mathrm{Ba}$ & 7,27 $\mathrm{ABa}$ & $4,66 \mathrm{Aa}$ & $5,34 \mathrm{Aa}$ & $13,02 \mathrm{Ba}$ & $12,60 \mathrm{Aa}$ & $64 \mathrm{Ca}$ & $58 \mathrm{Bb}$ & $31,12 \mathrm{Aa}$ & $11,10 \mathrm{Bb}$ \\
\hline \multirow[t]{2}{*}{ algodão/soja } & $10,91 \mathrm{Aa}$ & $6,62 \mathrm{Bb}$ & $2,04 \mathrm{Ba}$ & $2,80 \mathrm{Ba}$ & $12,95 \mathrm{Ba}$ & $9,43 \mathrm{~B}$ & $84 \mathrm{Aa}$ & $70 \mathrm{Ab}$ & $27,30 \mathrm{Aa}$ & $22,91 \mathrm{Aa}$ \\
\hline & \multicolumn{10}{|c|}{$10-20 \mathrm{~cm}$} \\
\hline \multirow[t]{2}{*}{ Área } & \multicolumn{2}{|c|}{$\mathrm{pH}\left(\mathrm{H}_{2} \mathrm{O}\right)$} & \multicolumn{2}{|c|}{$\mathrm{Ca}$} & \multicolumn{2}{|c|}{$\mathrm{Mg}$} & \multicolumn{2}{|c|}{$\mathrm{Na}$} & \multicolumn{2}{|c|}{$\mathrm{K}$} \\
\hline & $\mathrm{S}$ & $\mathrm{C}$ & $\mathrm{S}$ & $\mathrm{C}$ & $\mathrm{S}$ & $\mathrm{mol}_{\mathrm{c}} \mathrm{kg}^{-1} \mathrm{sc}$ & Slo------ & $\mathrm{C}$ & $\mathrm{S}$ & $\mathrm{C}$ \\
\hline Cerradão & 5,70 & 4,2 & 6,45 & 1,68 & 3,32 & 1,84 & 0,09 & 0,03 & 0,12 & 0,09 \\
\hline pasto/milho & $5,51 \mathrm{Ba}$ & $4,55 \mathrm{Bb}$ & 4,48 Aa & $2,77 \mathrm{Ab}$ & $3,65 \mathrm{Aa}$ & $2,22 \mathrm{ABb}$ & 0,088 Aa & $0,027 \mathrm{Ab}$ & $0,133 \mathrm{Ab}$ & $0,334 \mathrm{ABa}$ \\
\hline $\begin{array}{c}\text { milho+braquiária/ } \\
\text { algodão }\end{array}$ & $5,52 \mathrm{Ba}$ & $4,71 \mathrm{Bb}$ & $3,62 \mathrm{Aa}$ & $2,69 \mathrm{Aa}$ & 2,93 Aa & $2,43 \mathrm{Aa}$ & $0,087 \mathrm{Aa}$ & $0,022 \mathrm{Ab}$ & $0,179 \mathrm{Ab}$ & $0,427 \mathrm{Aa}$ \\
\hline algodão/soja & $6,47 \mathrm{Aa}$ & $5,07 \mathrm{Ab}$ & $4,63 \mathrm{Aa}$ & $2,78 \mathrm{Ab}$ & $3,02 \mathrm{Aa}$ & $1,59 \mathrm{Bb}$ & 0,089 Aa & $0,022 \mathrm{Ab}$ & $0,157 \mathrm{Ab}$ & $0,197 \mathrm{Ba}$ \\
\hline \multirow[t]{3}{*}{ Área } & \multirow{2}{*}{\multicolumn{2}{|c|}{ SB }} & \multicolumn{2}{|c|}{$\mathrm{H}+\mathrm{Al}$} & & $\mathrm{T}$ & & $\%$ & & $\mathrm{P}$ \\
\hline & & & $---\mathrm{cmol}_{\mathrm{c}}$ & $\mathrm{kg}^{-1}$ solo-- & --------. & ---------' & & & & $\mathrm{kg}^{-1}$ \\
\hline & $\mathrm{S}$ & $\mathrm{C}$ & $\mathrm{S}$ & $\mathrm{C}$ & $\mathrm{S}$ & $\mathrm{C}$ & $\mathrm{S}$ & $\mathrm{C}$ & $\mathrm{S}$ & $\mathrm{C}$ \\
\hline Cerradão & 9,97 & 3,64 & 6,64 & 9,24 & 16,62 & 12,88 & 60 & 28 & 1,26 & 0,02 \\
\hline pasto/milho & $8,36 \mathrm{Aa}$ & $5,35 \mathrm{Ab}$ & $4,29 \mathrm{Ab}$ & 5,36 Aa & $12,65 \mathrm{Aa}$ & $10,71 \mathrm{Ab}$ & $66 \mathrm{Ba}$ & $50 \mathrm{Bb}$ & $19,75 \mathrm{Aa}$ & $7,78 \mathrm{Ab}$ \\
\hline $\begin{array}{c}\text { milho+braquiária/ } \\
\text { algodão }\end{array}$ & $6,82 \mathrm{Ba}$ & $5,37 \mathrm{Ab}$ & 4,33 Aa & $4,73 \mathrm{Aa}$ & $11,15 \mathrm{Ba}$ & $10,30 \mathrm{Aa}$ & $61 \mathrm{Ba}$ & $54 \mathrm{Aba}$ & $12,46 \mathrm{Aa}$ & $4,22 \mathrm{Ab}$ \\
\hline algodão/soja & 7,90 ABa & $4,59 \mathrm{Ab}$ & $2,10 \mathrm{Ba}$ & $3,03 \mathrm{Ba}$ & $10,00 \mathrm{Ba}$ & 7,61 Bb & $79 \mathrm{Aa}$ & $60 \mathrm{Ab}$ & $11,63 \mathrm{Aa}$ & $6,51 \mathrm{Aa}$ \\
\hline & & & & & & $-30 \mathrm{~cm}$ & & & & \\
\hline & $\mathrm{pH}$ & $\left.\mathrm{H}_{2} \mathrm{O}\right)$ & & $\overline{\mathrm{Ca}}$ & & $\mathrm{Mg}$ & & $\mathrm{Na}$ & & $\mathrm{K}$ \\
\hline & $\mathrm{S}$ & $\mathrm{C}$ & $\mathrm{S}$ & $\mathrm{C}$ & $\mathrm{S}$ & $\mathrm{C}$ & $\mathrm{S}$ & $\mathrm{C}$ & $\mathrm{S}$ & $\mathrm{C}$ \\
\hline Cerradão & 5,59 & 3,98 & 4,57 & 1,28 & 3,03 & 1,68 & 0,090 & 0,02 & 0,11 & 0,08 \\
\hline pasto/milho & $5,48 \mathrm{Ba}$ & $4,42 \mathrm{Bb}$ & 4,03 Aa & $2,64 \mathrm{Ab}$ & $2,57 \mathrm{Aa}$ & $3,15 \mathrm{Aba}$ & $0,089 \mathrm{Aa}$ & $0,020 \mathrm{Bb}$ & $0,108 \mathrm{Ab}$ & $0,179 \mathrm{ABa}$ \\
\hline $\begin{array}{c}\text { milho+braquiária/ } \\
\text { algodão }\end{array}$ & $5,49 \mathrm{Ba}$ & $4,55 \mathrm{AB} b$ & $2,95 \mathrm{Ba}$ & $2,28 \mathrm{Aa}$ & $2,15 \mathrm{Aa}$ & $2,68 \mathrm{Ba}$ & 0,090 Aa & $0,043 \mathrm{Ab}$ & $0,144 \mathrm{Ab}$ & $0,216 \mathrm{Aa}$ \\
\hline algodão/soja & $6,22 \mathrm{Aa}$ & $4,87 \mathrm{Ab}$ & $3,60 \mathrm{ABa}$ & $2,23 \mathrm{Ab}$ & $2,32 \mathrm{Ab}$ & $3,59 \mathrm{Aa}$ & 0,091 Aa & $0,020 \mathrm{Bb}$ & $0,134 \mathrm{Aa}$ & $0,143 \mathrm{Ba}$ \\
\hline & & B & & $+\mathrm{Al}$ & & $\mathrm{T}$ & & $\%$ & & $\mathrm{P}$ \\
\hline & & & $---\mathrm{cmol}_{\mathrm{c}}$ & $\mathrm{kg}^{-1}$ solo- & ----- & ---------- & & & & $\mathrm{kg}^{-1}$ \\
\hline & $\mathrm{S}$ & $\mathrm{C}$ & $\mathrm{S}$ & $\mathrm{C}$ & $\mathrm{S}$ & $\mathrm{C}$ & $\mathrm{S}$ & $\mathrm{C}$ & $\mathrm{S}$ & $\mathrm{C}$ \\
\hline Cerradão & 7,80 & 3,06 & 6,94 & 8,77 & 14,74 & 11,83 & 51 & 26 & 2,28 & 0,22 \\
\hline pasto/milho & $6,80 \mathrm{Aa}$ & 5,99 Aa & 4,55 Aa & $5,14 \mathrm{Aa}$ & $11,35 \mathrm{Aa}$ & $11,13 \mathrm{Aa}$ & $59 \mathrm{Ba}$ & $54 \mathrm{Ba}$ & $5,45 \mathrm{Aa}$ & $2,62 \mathrm{Ab}$ \\
\hline $\begin{array}{c}\text { milho+braquiária/ } \\
\text { algodão }\end{array}$ & $5,33 \mathrm{Aa}$ & $5,21 \mathrm{Aa}$ & $4,08 \mathrm{Aa}$ & 4,43 Aa & $9,42 \mathrm{Ba}$ & $9,64 \mathrm{ABa}$ & $56 \mathrm{Ba}$ & $54 \mathrm{Ba}$ & $5,21 \mathrm{Aa}$ & $0,89 \mathrm{Ab}$ \\
\hline algodão/soja & $6,14 \mathrm{Aa}$ & $5,98 \mathrm{Aa}$ & $2,30 \mathrm{Ba}$ & $3,06 \mathrm{Aa}$ & $8,44 \mathrm{Ba}$ & $9,04 \mathrm{Ba}$ & $72 \mathrm{Aa}$ & $66 \mathrm{Aa}$ & $4,55 \mathrm{Aa}$ & $2,08 \mathrm{Ab}$ \\
\hline
\end{tabular}

Valores seguidos por letras maiúsculas iguais na coluna e minúsculas iguais na linha não diferem significativamente pelo teste $\mathrm{t}$ de Bonferroni a 5\%. S: época seca (maio/2009) e C: época chuvosa (março/2010). SB: soma de bases; T: capacidade de troca catiônica do solo; V\%: Porcentagem de saturação por bases.

Fonte: Elaboração dos autores. 
O estoque de $\mathrm{C}$ org e das frações granulométricas foi calculado pelo método de massa equivalente (ELLERT; BETTANY, 1995; SISTI et al.2004), conforme equação abaixo.

$C_{S}=\sum_{i=1}^{n-1} C_{T i}+\left[M_{T n}-\left(\sum_{i=1}^{n} M_{T i}-\sum_{i=1}^{n} M_{S i}\right)\right] C_{T n}$

onde: $C_{S}$ é o estoque total em $\mathrm{Mg} \mathrm{C}$ ha $^{-1}$,

$\sum_{i=1}^{n-1} C_{T i}$ é a soma do carbono da primeira (superfície) a última camada no perfil do solo no tratamento avaliado $\left(\mathrm{Mg} \mathrm{ha}^{-1}\right)$,

$\sum_{i=1}^{n} M_{T i}$ é a soma da massa do solo da primeira a última camada no perfil do solo no tratamento avaliado $\left(\mathrm{Mg} \mathrm{ha}^{-1}\right)$,

$\sum_{i=1}^{n} M_{S i}$ é a soma da massa do solo da primeira a última camada no perfil do solo no tratamento referência $\left(\mathrm{Mg} \mathrm{ha}^{-1}\right)$,

$M_{T n}$ é a massa do solo na última camada do perfil do solo no tratamento avaliado $\left(\mathrm{Mg} \mathrm{ha}^{-1}\right)$,

$C_{T n}$ é a concentração de carbono na última camada do perfil do tratamento avaliado $\left(\mathrm{Mg} \mathrm{C} \mathrm{Mg}^{-1} \mathrm{de}\right.$ solo).

A área de Cerradão não foi comparada com as áreas produtivas, visto que é um sistema natural e será utilizado somente como referência. Os dados obtidos foram avaliados quanto à normalidade (Teste de Lilliefors) e homogeneidade das variâncias dos erros (Teste de Cochran \& Barttlet) no programa estatístico SAEG 9.1 (2007). Em seguida, os dados foram submetidos à análise de variância com aplicação do Teste $\mathrm{t}$ (Bonferroni) a $5 \%$ de probabilidade no programa estatístico SISVAR 5.3 (FERREIRA; SISVAR, 2008).

\section{Resultados e Discussão}

No Tabela 3 são apresentados os teores de C org, COp e COam, nas profundidades 0-10, 10-20 e 20$30 \mathrm{~cm}$. O teor de $\mathrm{C}$ org variou de $37,57 \mathrm{~g} \mathrm{~kg}^{-1}$ a 24,90 de $0-10 \mathrm{~cm}$, de 27,80 a 13,49 em 10-20 cm e de 25,45 a 11,96 em 20-30 cm, nas áreas de Cerradão e algodão/soja respectivamente em todas as profundidades na época seca de avaliação. Ocorreu decréscimo dos teores de $\mathrm{C}$ org em profundidade, refletindo a deposição superficial dos resíduos e a maior concentração de raízes (CONCEIÇÃO et al., 2005; SALTON et al., 2005; SILVA et al., 2011).

Não foram observadas diferenças significativas nos teores de $\mathrm{C}$ org entre áreas e épocas de coleta, na profundidade de $0-10 \mathrm{~cm}$, o que pode ser atribuído ao constante aporte de resíduos vegetais. A área de algodão/soja apresentou menores teores de $\mathrm{C}$ org nas duas épocas de avaliação nas profundidades de 10-20 e 20-30, possivelmente por apresentar textura mais arenosa que as demais (Tabela 4), o que torna o material orgânico mais exposto ao ataque microbiano (PILON, MIELNICZUK; MARTIN NETO, 2002). Na Tabela 5 são apresentadas as principais correlações entre os atributos químicos e físicos do solo com os diferentes compartimentos da MOS. Pode-se observar que a fração areia apresentou correlação negativa com os diferentes compartimentos daMOS, enfatizando assim, a menor proteção da MOS aos agentes de decomposição, o que resulta em menores teores de C. Para a fração argila verificou-se correlação positiva, assim como para o valor $\mathrm{T}$. 
Tabela 3. Teores de carbono orgânico (C org), carbono orgânico particulado (COp) e carbono orgânico associado aos minerais (COam) nas profundidades 0-10, 10-20 e 20-30 cm.

\begin{tabular}{|c|c|c|c|c|c|c|}
\hline \multirow[t]{3}{*}{ Área } & \multicolumn{2}{|c|}{ C org } & \multicolumn{2}{|c|}{$\mathrm{COp}$} & \multicolumn{2}{|r|}{ COam } \\
\hline & \multicolumn{6}{|c|}{ 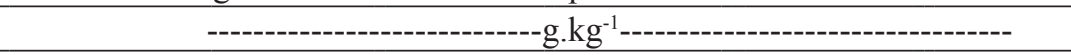 } \\
\hline & \multicolumn{6}{|c|}{$0-10 \mathrm{~cm}$} \\
\hline & $\mathrm{S}$ & $\mathrm{C}$ & $\mathrm{S}$ & $\mathrm{C}$ & $\mathrm{S}$ & $\mathrm{C}$ \\
\hline Cerradão & 37,57 & 33,9 & 20,22 & 8,32 & 17,35 & 25,6 \\
\hline pasto/milho & $27,85 \mathrm{Aa}$ & $25,2 \mathrm{Aa}$ & $18,37 \mathrm{Aa}$ & $7,81 \mathrm{Ab}$ & $9,48 \mathrm{Bb}$ & $17,4 \mathrm{Aa}$ \\
\hline milho+braquiária/algodão & $26,54 \mathrm{Aa}$ & $26,0 \mathrm{Aa}$ & $9,55 \mathrm{Ba}$ & $3,88 \mathrm{Bb}$ & $16,99 \mathrm{Aa}$ & $22,10 \mathrm{Aa}$ \\
\hline \multirow[t]{3}{*}{ algodão/soja } & $24,90 \mathrm{Aa}$ & $20,8 \mathrm{Aa}$ & $5,08 \mathrm{Ba}$ & $2,95 \mathrm{Ba}$ & $19,82 \mathrm{Aa}$ & $17,90 \mathrm{Aa}$ \\
\hline & $10-20 \mathrm{~cm}$ & & & & & \\
\hline & $\mathrm{S}$ & $\mathrm{C}$ & $\mathrm{S}$ & $\mathrm{C}$ & $\mathrm{S}$ & $\mathrm{C}$ \\
\hline Cerradão & 27,80 & 22,9 & 7,05 & 4,76 & 20,74 & 18,16 \\
\hline pasto/milho & $17,15 \mathrm{Aa}$ & $16,3 \mathrm{Ba}$ & $5,40 \mathrm{Aa}$ & $3,98 \mathrm{Aa}$ & $11,75 \mathrm{Ba}$ & $12,40 \mathrm{Ba}$ \\
\hline milho+braquiária/algodão & $17,86 \mathrm{Ab}$ & $20,9 \mathrm{Aa}$ & $2,25 \mathrm{Aa}$ & $2,28 \mathrm{Aa}$ & $15,61 \mathrm{Ab}$ & $18,68 \mathrm{Aa}$ \\
\hline \multirow[t]{3}{*}{ algodão/soja } & $13,49 \mathrm{Ba}$ & $14,1 \mathrm{Ba}$ & $2,09 \mathrm{Aa}$ & $5,93 \mathrm{Aa}$ & $11,40 \mathrm{Ba}$ & $12,32 \mathrm{Ba}$ \\
\hline & $20-30 \mathrm{~cm}$ & & & & & \\
\hline & $\mathrm{S}$ & $\mathrm{C}$ & $\mathrm{S}$ & $\mathrm{C}$ & $\mathrm{S}$ & $\mathrm{C}$ \\
\hline Cerradão & 25,45 & 22,30 & 4,66 & 5,19 & 20,79 & 17,12 \\
\hline pasto/milho & $14,80 \mathrm{Ab}$ & $17,57 \mathrm{Aa}$ & $4,85 \mathrm{Aa}$ & 3,77 Aa & $9,95 \mathrm{Ab}$ & $13,79 \mathrm{ABa}$ \\
\hline milho+braquiária/algodão & $13,93 \mathrm{ABa}$ & $16,12 \mathrm{Aa}$ & $1,43 \mathrm{Aa}$ & $6,64 \mathrm{Aa}$ & $12,49 \mathrm{Aa}$ & $14,00 \mathrm{Aa}$ \\
\hline algodão/soja & $11,96 \mathrm{Ba}$ & $12,3 \mathrm{Ba}$ & $1,51 \mathrm{Aa}$ & $1,17 \mathrm{Aa}$ & $10,45 \mathrm{Aa}$ & $11,13 \mathrm{Ba}$ \\
\hline
\end{tabular}

Valores seguidos por letras maiúsculas iguais na coluna e minúsculas iguais na linha não diferem significativamente pelo teste t de Bonferroni a 5\%. S: época seca e C: época chuvosa.

Fonte: Elaboração dos autores.

Os teores de COp variaram de 20,22 a 5,08 g $\mathrm{kg}^{-1}$ a 0-10 cm, de 7,05 a 2,09 $\mathrm{g} \mathrm{kg}^{-1}$ a $10-20 \mathrm{~cm}$, respectivamente, nas áreas de Cerradão e algodão/ soja e de 4,85 na área de pasto/milho a 1,43 na área de milho+braquiária/algodão de $20-30 \mathrm{~cm}$ na época seca. Assim como o C org, o COp também apresentou decréscimo dos teores em profundidade na época seca. Resultados semelhantes foram encontrados por Balbinot Júnior et al. (2011) em estudo de integração lavoura-pecuária e pousio no Planalto de Santa Catarina. Os autores verificaram que os teores $\mathrm{C}$ org e o COp foram maiores na camada superficial em comparação com as duas camadas abaixo, devido ao acúmulo de palha na superfície e a perturbação mínima do solo.
Houve redução dos teores de COp na época chuvosa na profundidade de $0-10 \mathrm{~cm}$ quando comparada à época seca nas áreas de pasto/milho e milho+braquiária/algodão (Tabela 3). Esse padrão de redução entre épocas pode ser explicado pela influência da maior precipitação nessa época associada às maiores temperaturas. A velocidade de decomposição da MOS está diretamente relacionada a esses fatores, que atuam na atividade de organismos decompositores (KHATOUNIAN, 1999). O COp é a fração da MOS que pode ser rapidamente decomposta, assim, uma frágil reserva $\mathrm{C}$ no solo e a diminuição dessa fração de $\mathrm{C}$ pode significar aumento das emissões de $\mathrm{CO}_{2}$ (FIGUEIREDO; RESCK; CARNEIRO, 2010). 
Tabela 4. Teores de areia, silte e argila das amostras de terra nas profundidades 0-10, 10-20 e 20-30 cm.

\begin{tabular}{|c|c|c|c|}
\hline \multicolumn{4}{|c|}{$0-10 \mathrm{~cm}$} \\
\hline Área & Areia & Silte & Argila \\
\hline Cerradão & 173 & 275 & 552 \\
\hline pasto/milho & 209 & 230 & 560 \\
\hline milho+braquiária/algodão & 465 & 131 & 404 \\
\hline algodão/soja & 560 & 133 & 307 \\
\hline \multicolumn{4}{|c|}{$10-20 \mathrm{~cm}$} \\
\hline Área & Areia & Silte & Argila \\
\hline & & ------ & --- \\
\hline Cerradão & 157 & 271 & 572 \\
\hline pasto/milho & 189 & 258 & 553 \\
\hline milho+braquiária/algodão & 459 & 131 & 410 \\
\hline algodão/soja & 540 & 120 & 340 \\
\hline \multicolumn{4}{|c|}{$20-30 \mathrm{~cm}$} \\
\hline Área & Areia & Silte & Argila \\
\hline Cerradão & 170 & 257 & 571 \\
\hline pasto/milho & 189 & 245 & 566 \\
\hline milho+braquiária/algodão & 442 & 130 & 428 \\
\hline algodão/soja & 571 & 85 & 344 \\
\hline
\end{tabular}

Fonte: Elaboração dos autores.

Tabela 5. Matriz de correlação entre os principais parâmetros utilizados nas épocas seca e chuvosa de avaliação.

\begin{tabular}{ccccccc}
\hline \multicolumn{7}{c}{ Época seca } \\
\hline Areia & C org & COp & COam & EstCorg & EstCOp & EstCOam \\
\hline Silte & $-0,44^{*}$ & $-0,49^{* *}$ & $-0,18$ & $-0,51^{* *}$ & $-0,59^{* *}$ & $-0,19$ \\
Argila & $0,51^{* *}$ & $0,48^{* *}$ & 0,33 & $0,53^{* *}$ & $0,71^{* *}$ & $-0,29$ \\
SB & 0,37 & $0,47^{* *}$ & 0,07 & $0,44^{*}$ & $0,57^{* *}$ & 0,09 \\
T & $0,88^{* *}$ & $0,83^{* *}$ & $0,58^{* *}$ & $0,85^{* *}$ & $0,81^{* *}$ & $0,55^{* *}$ \\
V\% & $0,93^{* *}$ & $0,82^{* *}$ & $0,67^{* *}$ & $0,93^{* *}$ & $0,86^{* *}$ & $0,64^{* *}$ \\
\hline \multicolumn{1}{c}{} & 0,18 & $-0,04$ & 0,02 & 0,09 & $-0,04$ \\
\hline Areia & 0,11 & Expoca chuvosa & & & \\
Silte & C org & COp & COam & EstCorg & EstCOp & EstCOam \\
Argila & $-0,41^{*}$ & $-0,49^{* *}$ & $-0,18$ & $-0,49^{* *}$ & $-0,58^{* *}$ & $-0,31$ \\
SB & $0,47^{*}$ & $0,46^{*}$ & 0,24 & $0,54^{* *}$ & $0,55^{* *}$ & 0,35 \\
T & 0,36 & $0,49^{*}$ & 0,13 & $0,44^{*}$ & $0,58^{* *}$ & 0,27 \\
V\% & 0,11 & $-0,07$ & 0,11 & $-0,01$ & $-0,21$ & 0,02 \\
\hline
\end{tabular}

Época seca: maio/2009; Época chuvosa: março/2010; * e** : significativos a 10 e 5 \%. C org: Carbono orgânico; COp: Carbono orgânico particulado; COam: Carbono orgânico associado aos minerais; EstCorg: Estoque de C org; EstCOp: Estoque de COp; EstCOam: Estoque de COam; SB: Soma de bases; T: capacidade de troca cationica do solo; V\%: Porcentagem de saturação por bases.

Fonte: Elaboração dos autores. 
Verificou-se que na fração COp, as mudanças provenientes das diferentes coberturas do solo foram mais sensíveis na profundidade de $0-10 \mathrm{~cm}$, em comparação com o $\mathrm{C}$ org que não apresentou diferença entre as áreas. Deste modo, observase que o $\mathrm{COp}$ pode funcionar como indicador da qualidade da MOS em relação às diferentes coberturas. A sensibilidade do COp também foi observada por Conceição et al. (2005) avaliando a qualidade do solo sob diferentes sistemas de manejo em experimentos de longa duração no sul do Brasil. Na profundidade de $0-5 \mathrm{~cm}$, os autores verificaram que através da sensibilidade da fração particulada da MOS, pode-se inferir que este compartimento possa ser utilizado como indicador de qualidade do solo para avaliação de sistemas de manejo recentes, nos quais as alterações no $\mathrm{C}$ org do solo ainda não tenham sido de grande magnitude.

O teor de COam apresentou grande participação no $\mathrm{C}$ org quando comparado com COp, na área de algodão/soja por exemplo, essa participação no $\mathrm{C}$ org na época chuvosa correspondeu a valores de $86 \%, 87 \%$ e $90 \%$ nas profundidades de $0-10$, $10-20$ e $20-30 \mathrm{~cm}$ respectivamente. Resultados semelhantes foram encontrados por Salton et al. (2005), que verificaram de maneira geral, valores entre $77 \%$ e $93 \%$ do COT correspondendo a fração associada aos minerais do solo. A menor participação da fração particulada nas camadas subsuperficiais do solo também foi observada por Nicoloso (2005) em estudo em áreas de ILP no RS, onde o autor verificou o aumento da participação do COam em profundidade, demonstrando também que a variação dos teores de COp entre as diferentes áreas estudadas, na profundidade de 0-5 cm, é dependente da adição de resíduos vegetais. O COp posteriormente vai contribuir no compartimento estável da MOS (associado aos minerais). Dessa forma, se existe elevada participação do COam no $\mathrm{C}$ org, significa que na área está ocorrendo grande estabilização do $\mathrm{C}$ e provavelmente diminuição da emissão de C. Contudo, é desejável que o solo apresente adequada quantidade de COp pois desta forma estará garantindo fluxo de C para o solo e assim mantendo sua atividade biológica. Por outro lado, caso o solo não disponha de MO lábil em quantidade suficiente para suprir suas necessidades, os processos de oxidação da MOS irão resultar em redução do estoque de $\mathrm{C}$ no solo, dando início ao processo de perda de qualidade e degradação do solo (SALTON et al., 2005)

Na Tabela 6 são apresentados os estoques C org, COp e COam, nas profundidades 0-10, 10-20 e 20-30 cm. Observa-se que o estoque de $\mathrm{C}$ org diminuiu em profundidade, resultados semelhantes foram encontrados por Salton et al. (2011) em áreas de ILP na região de Campo Grande, Dourados e Maracaju - MS, onde, os autores atribuem este fato ao acúmulo de resíduos na camada superficial do solo. Silva et al. (2011) estudando áreas de ILP no Cerrado após quatro e oito anos implantação, também encontraram resultados semelhantes, e atribuem este padrão ao maior aporte de resíduo em superfície e o revolvimento somente na linha de plantio.

$\mathrm{O}$ estoque de COp foi sensível às diferentes coberturas do solo nas profundidades de 0-10 e 10$20 \mathrm{~cm}$, a área de pasto/milho apresentou o maior estoque, no entanto, o teor desta fração apresentou diferenças em relação as demais áreas a $0-10 \mathrm{~cm}$ de profundidade no período seco. Esse resultado mostra a importância da quantificação do estoque de carbono na avaliação de diferentes coberturas do solo. O estoque de COam apresentou padrão inverso, foi sensível quanto aos teores, na época seca nas profundidades de $0-10$ e $10-20 \mathrm{~cm}$ e na época chuvosa nas profundidades de $10-20$ e 20$30 \mathrm{~cm}$, não sendo verificadas diferenças quanto ao estoque em profundidade. Bayer et al. (2004) estudaram quatro rotações em SPD, encontrando resultados semelhantes, que demonstraram que o estoque de COam não foi afetado pelos sistemas de manejo nas diferentes camadas de solo. Segundo os autores essa fração apresenta um avançado estádio de humificação e é altamente estável devido a sua interação com a fração mineral e localização no 
interior de microagregados estáveis. Nicoloso (2005) em estudo em áreas de ILP no RS também encontraram resultados semelhantes, para o autor, seria necessário que houvesse uma grande variação nas práticas de manejo e/ou cobertura vegetal para que fossem observadas diferenças no estoque dessa fração, o que pode não ocorrer em curtos períodos de avaliação, e/ou áreas onde a ciclagem de nutrientes ocorra de maneira mais lenta.

Tabela 6. Densidade do solo (Ds), estoque de carbono orgânico (C org) e nas frações particulada (COp) e associada aos minerais (COam) nas profundidades de $0-10,10-20$ e $20-30 \mathrm{~cm}$.

\begin{tabular}{|c|c|c|c|c|c|c|c|}
\hline \multirow{6}{*}{$\begin{array}{c} \\
\\
\text { Cerradão } \\
\text { pasto/milho } \\
\begin{array}{c}\text { milho+braquiária/algodão } \\
\text { algodão/soja }\end{array} \\
\end{array}$} & \multirow{3}{*}{$\begin{array}{c}\text { Ds } \\
\mathrm{Mg} \mathrm{m}^{-3} \\
105\end{array}$} & \multicolumn{2}{|c|}{ C org } & \multicolumn{2}{|c|}{$\mathrm{COp}$} & \multicolumn{2}{|c|}{ COam } \\
\hline & & \multicolumn{4}{|c|}{$0-10$} & & \\
\hline & & 42,06 & 33,75 & 20,27 & 7,59 & 21,80 & 26,16 \\
\hline & 1,66 & $29,99 \mathrm{Aa}$ & $25,43 \mathrm{Aa}$ & $17,63 \mathrm{Aa}$ & $7,89 \mathrm{Ab}$ & $12,37 \mathrm{Aa}$ & $17,54 \mathrm{Aa}$ \\
\hline & 1,60 & $30,25 \mathrm{Aa}$ & $26,75 \mathrm{Aa}$ & $12,34 \mathrm{Ba}$ & $3,57 \mathrm{Ab}$ & $17,91 \mathrm{Aa}$ & $23,18 \mathrm{Aa}$ \\
\hline & 1,56 & $26,79 \mathrm{Aa}$ & $23,08 \mathrm{Aa}$ & $5,39 \mathrm{Ca}$ & $3,45 \mathrm{Aa}$ & $21,40 \mathrm{Aa}$ & $19,63 \mathrm{Aa}$ \\
\hline & & & & $10-20$ & & & \\
\hline Cerradão & 1,12 & 34,54 & 27,84 & 16,87 & 7,88 & 17,67 & 19,96 \\
\hline pasto/milho & 1,59 & $19,17 \mathrm{Aa}$ & $17,24 \mathrm{Ba}$ & $5,95 \mathrm{Aa}$ & $4,87 \mathrm{Aa}$ & $13,22 \mathrm{Aa}$ & $12,37 \mathrm{Aa}$ \\
\hline milho+braquiária/algodão & 1,63 & $20,04 \mathrm{Ab}$ & $23,71 \mathrm{Aa}$ & 2,49 Ba & $3,28 \mathrm{ABa}$ & $13,22 \mathrm{Aa}$ & $12,37 \mathrm{Aa}$ \\
\hline algodão/soja & 1,68 & $15,41 \mathrm{Aa}$ & $14,89 \mathrm{Ba}$ & $2,25 \mathrm{Ba}$ & $1,41 \mathrm{Ba}$ & $13,16 \mathrm{Aa}$ & $13,47 \mathrm{Aa}$ \\
\hline & & & & $20-30$ & & & \\
\hline Cerradão & 1,15 & 30,84 & 24,96 & 6,26 & 5,96 & 24,58 & 19,00 \\
\hline pasto/milho & 1,48 & 17,39 Aa & $20,98 \mathrm{Aa}$ & $5,57 \mathrm{Aa}$ & 4,40 Aa & $11,83 \mathrm{Ab}$ & $16,58 \mathrm{Aa}$ \\
\hline milho+braquiária/algodão & 1,61 & $16,34 \mathrm{Aa}$ & $18,75 \mathrm{Aa}$ & $1,97 \mathrm{Aa}$ & $1,17 \mathrm{Aa}$ & $14,37 \mathrm{Aa}$ & $17,58 \mathrm{Aa}$ \\
\hline algodão/soja & 1,64 & $14,02 \mathrm{Aa}$ & $13,54 \mathrm{Ba}$ & 2,20 Aa & $1,34 \mathrm{Aa}$ & $11,82 \mathrm{Aa}$ & $12,20 \mathrm{Aa}$ \\
\hline
\end{tabular}

Valores seguidos por letras maiúsculas iguais na coluna e minúsculas iguais na linha não diferem significativamente pelo teste $\mathrm{t}$ de Bonferroni a 5\%. S: época seca (maio/2009) e C: época chuvosa (março/2010).

Fonte: Elaboração dos autores.

\section{Conclusões}

A área de pasto/milho proporcionou maior estoque de carbono na fração particulada na camada superficial do solo;

A área de algodão/soja, devido a sua textura mais arenosa, foi a que apresentou maiores perdas de carbono;

Devido às áreas possuírem o mesmo histórico, o estoque da fração mais humificada não se mostrou sensível às variações de cobertura;

A fração COp apresenta-se mais sensível às diferentes coberturas do solo e sazonalidade.

\section{Referências}

BALBINOT JÚNIOR, A. A.; VEIGA, M.; MORAES, A.; PELISSARI, A.; MAFRA, A. L.; PICCOLLA, C. D. Winter pasture and cover crops and their effects on soil and summer grain crops. Pesquisa Agropecuária Brasileira, Brasília, v. 46, n. 10, p. 1357-1363, out. 2011.

BAYER, C.; MARTIN-NETO, L.; MIELNICZUK, J.; PAVINATO, A. Armazenamento de carbono em frações lábeis da matéria orgânica de um Latossolo Vermelho sob plantio direto. Pesquisa Agropecuária Brasileira, Brasília, v. 39, n. 7, p. 677-683, 2004.

CAMBARDElla, C. A.; ELLIOTT, E. T. Particulate soil organic matter changes across a grassland cultivation sequence. Soil Science Society of America Journal, Madison, v. 56, n. 3, p. 777-783, 1992. 
CONCEIÇÃO, P. C.; AMADO, T. J. C.; MIELNICZUK, J.; SPAGNOLLO, E. Qualidade do solo em sistemas de manejo avaliada pela dinâmica da matéria orgânica e atributos relacionados. Revista Brasileira de Ciência do Solo, Viçosa, MG, v. 29, n. 5, p. 777-788, 2005.

COSTA, F. S.; BAYER, C.; ACORDI, Z.; MIELNICZUK, J. Estoque de carbono orgânico no solo e emissões de dióxido de carbono influenciadas por sistemas de manejo no sul do Brasil. Revista Brasileira de Ciência do Solo, Viçosa, MG, v. 32, n. 1, p. 323-332, 2008.

CRUZ, J. C.; ALVARENGA, R. C.; NOVOTNY, E. H.; PEREIRA-FILHO, I. A.; SANTANA, D. P.; PEREIRA, F. T. F.; HERNANI, L. C. Cultivo do milho. 2. ed. Sete Lagoas: Embrapa Milho e Sorgo, versão eletrônica, dez. 2006. 4 p. (Sistema de Produção, 1).

DAY, P. R. Particle fractionation and particle-size analysis. In: BLACK, C. A. (Ed.). Methods of soil analysis. Madison: American Society of Agronomy, 1965. v. 1, p. 545-556.

DORAN, J. W.; PARKIN, T. B. Defining and assessing soil quality. In: DORAN, J. W. COLEMAN, D. C., BEZDICEK, D. F.; STEWART, B. A. Defining soil quality for a sustainable environment. Madison: Soil Science Society of America, 1994. v. 35, p. 3-22.

ELLERT, B. H.; BETTANY, J. R. Calculation of organic matter and nutrients stored in soils under contrasting management regimes. Canadian Journal Soil Science, Ottawa, v. 75, n. 4, p. 529-538, 1995.

EMPRESA BRASILEIRA DE PESQUISA AGROPECUÁRIA - EMBRAPA. Centro Nacional de Pesquisa de Solos. Manual de métodos de análise de solos. 2. ed. Rio de Janeiro: EMBRAPA, 1997. 212 p.

Centro Nacional de Pesquisa de Solos. Sistema brasileiro de classificação de solos. 2. ed. Rio de Janeiro: EMBRAPA, 2006. 306 p.

FERREIRA, D. F. SISVAR: um programa para análises estatísticas e ensino de estatística. Revista Symposium, Lavras, v. 6, n. 1, p. 36-41, 2008.

FIGUEIREDO, C. C.; RESCK, D. V. S.; CARNEIRO, M.A. C. Labile and stable fractions of soil organic matter under management systems and native cerrado. Revista Brasileira de Ciência do Solo, Viçosa, MG, v. 34, n. 3, p. 907-916, 2010.

FRANZLUEBBERS, A, J. Soil organic matter stratification ratio as an indicator of soil quality. Soil \& Tillage Research, Amsterdam, v. 66, n. 2, p. 95-106, 2002.
FREIXO, A. A.; CANELLAS, L. P.; MACHADO, P. L. O. A. Propriedades espectrais da matéria orgânica levelivre e leve intra-agregado de dois latossolos sob plantio direto e preparo convencional. Revista Brasileira de Ciência do Solo, Viçosa, MG, v. 26, n. 2, p. 445-453, 2002a.

FREIXO, A. A.; MACHADO, P. L. O. A.; GUIMARÃES, C. M.; SILVA, C. A.; FADIGAS, F. S. Estoques de carbono e nitrogênio e distribuição de frações orgânicas de Latossolo do Cerrado sob diferentes sistemas de cultivo. Revista Brasileira de Ciência do Solo, Viçosa, MG, v. 26, n. 2, p. 425-434, 2002 b.

GREGORICH, E. G.; CARTER, M. R.; ANGERS, D. A.; MONREALL, C. M.; ELLERTA, B. H. Towards a minimum data set to assess soil organic matter quality in agricultural soils. Canadian Journal of Soil Science, Ottawa, v. 74, n. 4, p. 367-385, 1994.

INSTITUTO BRASILEIRO DE GEOGRAFIA E ESTATÍSTICA - IBGE. Indicadores de desenvolvimento sustentável. Estudos e pesquisas: informação geográfica 7. Rio de Janeiro: IBGE, 2010. p. 443.

KHATOUNIAN, C. A. O manejo da fertilidade em sistemas de produção. In: CASTRO FILHO, C.; MUZILLI, O. (Coord.). Uso e manejo dos solos de baixa aptidão agrícola. Londrina: IAPAR, 1999. p. 179-221. (Circular, 108).

MACEDO, M. C. M. Integração lavoura e pecuária: o estado da arte e inovações tecnológicas. Revista Brasileira de Zootecnia, Viçosa, MG, v. 38, p. 133-146, 2009. Suplemento Especial.

NICOLOSO, R. S. Dinâmica da matéria orgânica do solo em áreas de integração lavoura-pecuária sob sistema plantio direto. 2005. Dissertação (Mestrado em Ciência do Solo) - Universidade Federal de Santa Maria, Santa Maria.

PILON, C. N.; MIELNICZUK, J.; MARTIN NETO, L. Dinâmica da matéria orgânica no ambiente. Pelotas: Embrapa Clima Temperado, 2002. 41 p. (Embrapa Clima Temperado. Documentos, 105).

RESK, D. V. S.; FERRREIRA, E. A. B.; FIGUEIREDO, C. C.; ZINN, Y. L. Dinâmica da matéria orgânica no Cerrado. In: SANTOS, G. A.; SILVA, L. S.; CANELLAS, L. P.; CAMARGO, F. A. O. Fundamentos da matéria orgânica do solo: ecossistemas tropicais e subtropicais. 2. ed. Porto Alegre: Editora Cinco Continentes, 2008, p. 359-417. 
SALTON, J. C.; MIELNICZUK, J.; BAYER, C.; FABRICIO, A. C.; MACEDO, M. C. M.; BROCH, D. L.; BOENI, M.; CONCEIÇÃO, P. C. Matéria orgânica do solo na integração lavoura-pecuária em Mato Grosso do Sul. Dourados: Embrapa Agropecuária Oeste, 2005. 58 p. (Embrapa Agropecuária Oeste, Boletim de Pesquisa e Desenvolvimento, 29).

SALTON, J. C.; MIELNICZUK, J.; BAYER, C.; FABRÍCIO, A. C.; MACEDO, M. C. M.; BROCH, D. L. Teor e dinâmica do carbono no solo em sistemas de integração lavoura-pecuária. Pesquisa Agropecuária Brasileira, Brasília, v. 46, n. 10, p. 1349-1356, out. 2011.

SILVA, J. E.; RESK, D. V. S.; CORAZZA, E. J.; VIVALDI, L. Carbon storage in clayey Oxisol cultivated pastures in the "Cerrado" region, Brazil. Agriculture, Ecosystems and Environment, Amsterdam, v. 103, n. 2, p. 357-363, 2004.

SILVA, E. F.; LOURENTE, E. P. R.; MARCHETTI, M. E.; MERCANTE, F. M.; FERREIRA, A. C. T.; FUJII, G. C. Frações lábeis e recalcitrantes da matéria orgânica em solos sob integração lavoura-pecuária. Pesquisa Agropecuária Brasileira, Brasília, v. 46, n. 10, p. 13211331, out. 2011.

SISTEMA PARA ANÁLISES ESTATÍSTICAS - SAEG versão 9.1.Viçosa: Fundação Arthur Bernardes, UFV, 2007.
SISTI, C. P. J.; SANTOS, H. P.; KOHHANN, R.; ALVES, B. J. R.; URQUIAGA, S.; BODDEY, R. M. Change in carbon and nitrogen stocks in soil under 13 years of conventional or zero tillage in southern Brazil. Soil and Tillage Research, Amsterdam, v. 76, n. 1, p. 39-58, 2004.

SOUZA, E. D.; COSTA, S. E. V. G. A.; ANGHINONI, I.; CARVALHO, P. C. F.; ANDRIGUETI, M.; CAO, E. Estoques de carbono orgânico e de nitrogênio no solo em sistema de integração lavoura-pecuária em plantio direto, submetido a intensidades de pastejo. Revista Brasileira de Ciência do Solo, Viçosa, MG, v. 33, n. 6, p. 18291836, 2009.

VILELA, L.; MARTHA JUNIOR, G. B.; MACEDO, M. C.; MARCHÃO, R. L.; GUIMARÃES JUNIOR, R.; PULROLNIK, K.; MACIEL, G. A. Sistemas de integração lavoura-pecuaria na região do Cerrado. Pesquisa Agropecuária Brasileira, Brasília, v. 46, n. 10, p. 1127-1138, out. 2011.

YEOMANS, J. C.; BREMNER, J. M. A rapid and precise method for routine determination of organic carbon in soil. Communications in Soil Science and Plant Analysis, London, v. 19, n. 13, p. 1467-1476, 1988. 